\title{
A porcupine Sun? Implications for the solar wind and Earth
}

\author{
Sarah E. Gibson ${ }^{1}$ and Liang Zhao ${ }^{1}$ \\ ${ }^{1}$ NCAR/HAO, 3080 Center Green Dr., Boulder, CO \\ email: sgibson@ucar .edu
}

\begin{abstract}
The recent minimum was unusually long, and it was not just the case of the "usual story" slowed down. The coronal magnetic field never became completely dipolar as in recent Space Age minima, but rather gradually evolved into an (essentially axisymmetric) global configuration possessing mixed open and closed magnetic structures at many latitudes. In the process, the impact of the solar wind at the Earth went from resembling that from a sequence of rotating "fire-hoses" to what might be expected from a weak, omnidirectional "lawn-sprinkler". The previous (1996) solar minimum was a more classic dipolar configuration, and was characterized by slow wind of hot origin localized to the heliospheric current sheet, and fast wind of cold origin emitted from polar holes, but filling most of the heliosphere. In contrast, the more recent minimum solar wind possessed a broad range of speeds and source temperatures (although cooler overall than the prior minimum). We discuss possible connections between these observations and the near-radial expansion and small spatial scales characteristic of the recent minimum's porcupine-like magnetic field.
\end{abstract}

Keywords. Sun: solar-terrestrial relations, Sun: solar wind; Sun: corona, Sun: magnetic fields

\section{Introduction}

The Whole Heliosphere Interval (WHI) (Thompson et al. 2011) was an internationally coordinated observing and modeling effort. Its science goals were to connect the origins and effects of solar structure and activity through the solar wind to the Earth and other planetary systems, and to characterize the three-dimensional solar minimum heliosphere. Results from WHI may be found in the 2011 Topical Issue of Solar Physics: "The SunEarth Connection near Solar Minimum."

In this paper, we will draw from analyses within that Topical Issue and elsewhere in order to summarize the evolution of the recent solar minimum, and compare it to the one immediately prior. In particular, we will discuss three representative properties: the global coronal magnetic field, the global solar wind structure in relation to the Earth's space environment, and the solar wind source temperature as encoded in its composition.

\section{Comparing solar minima: Coronal magnetic field and solar wind/Earth interactions}

The most recent minimum was different from the previous two minima, and the beginning of the recent minimum was unlike its end. In an overview article of the Solar Physics WHI Topical Issue, Gibson et al. (2011) described the prolonged recent minimum from Sun to Earth as it evolved from 2008 through 2009. In terms of magnetic flux emergence and associated closed-field quanitites (e.g., sunspots, solar irradiance, coronal mass ejections) there was not much change during these years, with levels essentially staying as low or lower than the prior minimum. However, for quantities pertaining to or sensitive to the distribution of open flux at the Sun and in the heliosphere (e.g., coronal 


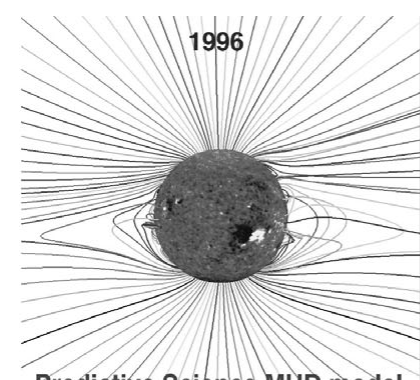

Predictive Science MHD model
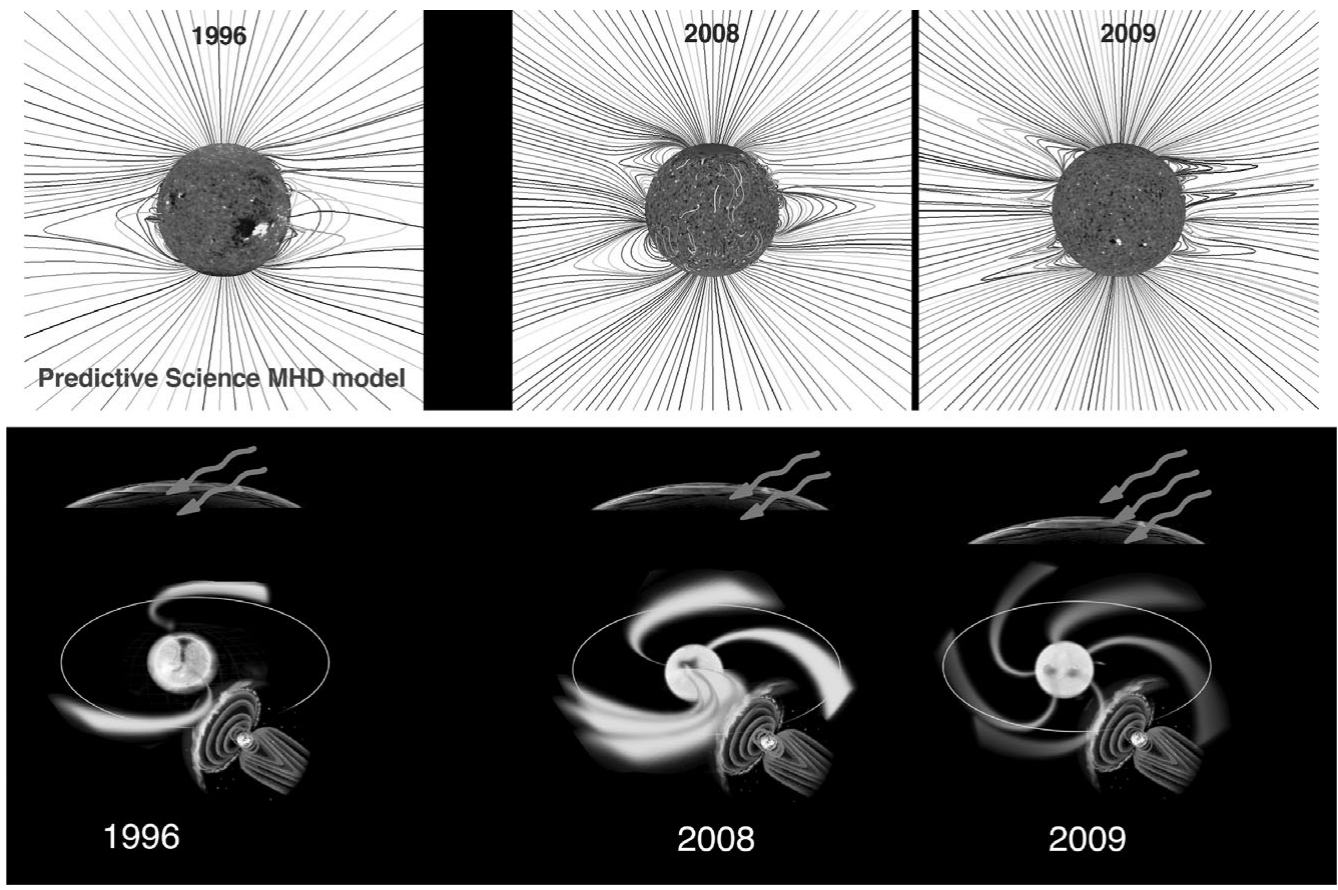

Figure 1. Top: Coronal magnetic field from the Magnetohydrodynamics on a Sphere (MAS) model for (left to right) Whole Sun Month Carrington Rotation (CR) 1913 in August-September 1996, August 1, 2008 eclipse prediction (using CR2071 and CR2072 data), and July 22, 2009 eclipse (using CR2084 and CR2085). Bottom: artist's conception for similar times of solar wind morphology (faster wind streams indicated in yellow emanating from coronal holes) and impact for the Earth's radiation belt (large relativistic electron population indicated by red) and cosmic rays (high levels indicated by number of squiggly red arrows). For color figures, see the online version of this paper.

holes, heliospheric current sheet (HCS) tilt, cosmic rays, solar wind velocity at the Earth, the Earth's radiation belts) there was significant evolution, from levels that had not yet reached those of the prior solar minimum in early 2008, to space-age-record-setting levels by mid 2009 .

Figure 1 (top row) illustrates the magnetic morphology of the corona. Last minimum reached a predominantly dipole state, with closed field straddling a fairly flat HCS. 2008 saw a warped HCS, and both open and closed field at a broader range of latitudes. In 2009 the HCS flattened, but because of a weak polar magnetic field the dipole was not as dominant as in 1996. Both closed and open field were still present at low-to-mid latitudes, although the non-polar open-field coronal holes were no longer large and longlived as they had been in 2008 (de Toma 2011). Moreover, much of the closed field in both 2008 and 2009 were so-called "pseudo" streamers, in that they were surrounded by open magnetic field of a single polarity (Riley \& Luhmann 2012). The HCS-straddling closed field of the more traditional streamer was still present as it was in 1996, but, particularly in 2009, was narrower in latitudinal width, as were also the other pseudostreamer closed field regions.

Figure 1 (bottom row) shows an artist's conception of the solar wind for these time periods. During 1996, the Sun was not completely dipolar in that there were equatorward extensions of the Northern polar hole, which drove the Earth's space environment with fast solar wind streams for several months as they rotated past. However, as discussed in 
Gibson et al. (2009), the presence of large, long-lived and low-latitude coronal holes in 2008 had a much stronger effect, leading to periodicities and elevated levels in the Earth's radiation belts and aurora (also the thermosphere, as discussed in Lei et al. (2010) and Gibson et al. (2011)). By 2009 those coronal holes had dissipated, and the Sun's magnetic configuration was essentially axiymmetric. It was still complex, with open and narrowclosed "porcupine"-like magnetic structures poking out in all directions, but the loss of the localized long-lived open field structures resulted in a loss of the periodic forcing of fast wind streams and associated enhanced radiation belts. Overall, the heliosphere reached the depths of its minimum, and cosmic rays were free to reach space-age record high levels, due at least in part to space-age record-low levels of interplanetary magnetic field strength (Mewaldt et al. 2010).

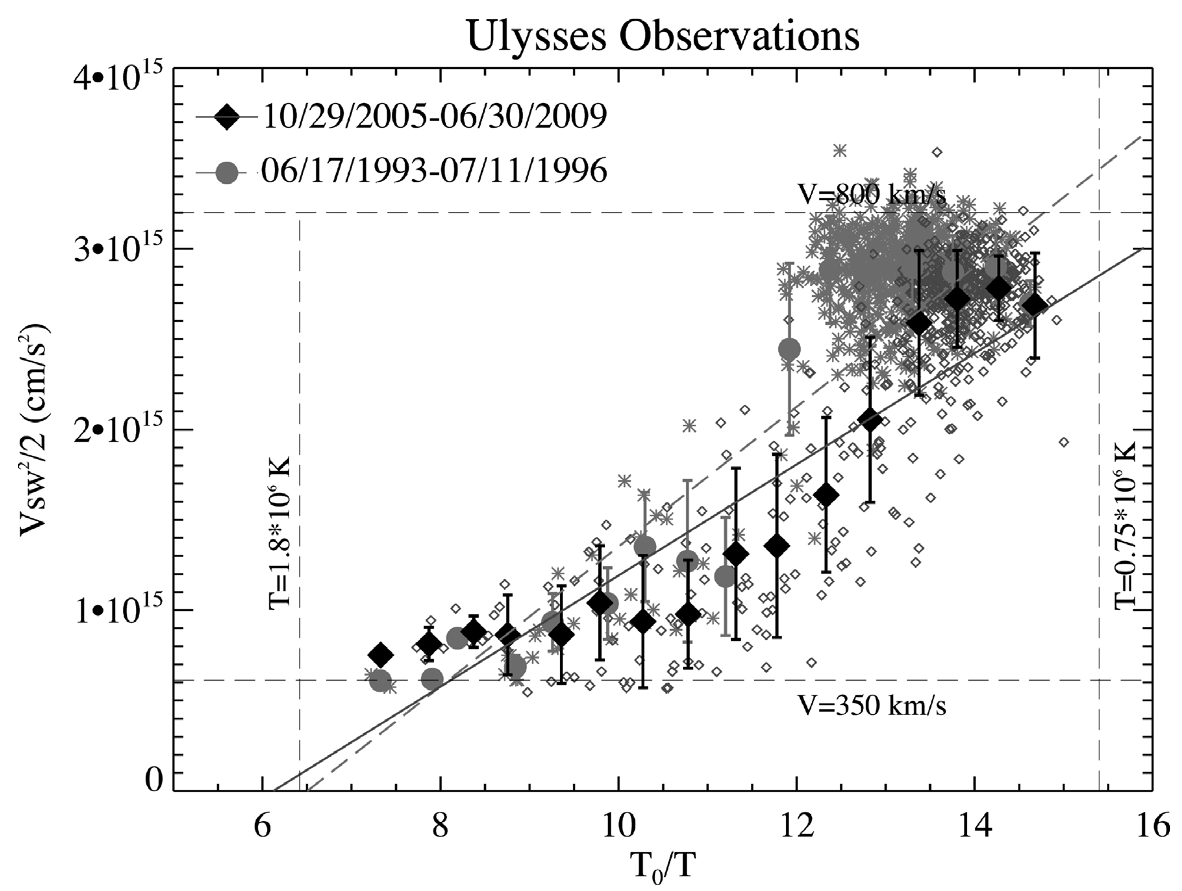

Figure 2. Ulysses measurements of solar wind source temperature $(T)$ extracted from Oxygen charge-state ratio. $T_{o} / T$ is plotted vs. solar wind velocity-squared, where $T_{o} \simeq 14 M K\left(T_{o}\right.$ is defined in Zhao \& Fisk (2011) and has a weak dependence on temperature - see Fig. 13 of that paper for a comparable plot). The black diamonds are binned (bin size: $T_{0} / T=0.5$ ) averages for the most recent minimum (shown with higher resolution by smaller blue diamonds), and the red large dots are the binned averages for the prior minimum (shown with higher resolution by the smaller red asterisks). The time intervals are of the same length and Ulysses orbital position for the two minima. ICMEs have been removed by eliminating points with high values of $O^{7+} / O^{6+}$. For color figures, see the online version of this paper.

\section{Comparing solar minima: Solar wind composition}

The solar magnetic field morphologies of the last two minima were also reflected in solar wind composition. For both minima the solar wind $O^{7+} / O^{6+}$ ratio, related to the temperature of solar wind source regions, indicated slow wind from a hot source at HCS crossings, and fast wind from a cold source deep in polar holes. However, just as the closed field around the HCS was narrower in the more recent minimum, Zhao \& Fisk 
(2011) found that the width in the heliosphere of the slow, hot-sourced wind straddling the HCS was narrower.

Figure 2 shows the source temperature extracted from the Oxygen charge-state ratio plotted versus velocity-squared, illustrating an inverse correlation that ranges from hotsourced, slow wind to cold-sourced, fast wind. To first order this relationship has not changed greatly from one minimum to the other. Note that the time intervals chosen are for the same Ulysses orbital positions and similar solar cycle levels.

However, a closer look reveal two interesting differences between the two minima. First, there is a general shift to the right in Figure 2 from the 1996 minimum (red/asterisks) to the more recent minimum (blue/diamonds). This indicates a a cooler source during the recent minimum, particularly for the slow, cold-sourced wind (upper right) coming from the polar coronal holes (von Steiger \& Zurbuchen 2011).

Second, it is clear that the distribution in the most recent minimum is not as bimodal as the prior minimum. At times near the 1996 minimum, the heliosphere was largely filled with fast, cold-polar-hole-sourced wind, as is evidenced by the large clump of red points in the upper right of Figure 2. As the Ulysses orbit crossed the HCS, some slow, hot-sourced wind was seen (bottom left), but there are not many red points in between these two extremes. In contrast, the most recent minimum possessed a broad range of source temperatures and velocities, as seen in the more uniformly-spaced curve of blue dots.

\section{Discussion and Conclusions}

The magnetic field at the solar surface controls the distribution of open flux in the corona, the morphology and temperature of the coronal plasma, and the structure and impact of the solar wind at the Earth. The differences between the two solar minima were reflected in all of these properties.

The solar wind Oxygen composition indicates that, although the behavior in the depths of the coronal hole or right at the HCS were similar for the two minima, a population of warm/mid-speed wind was also present in the more recent minimum. It seems likely that this was related to the population of mixed closed/open field seen at mid-to-low latitudes in the recent minimum, and, in particular, pseudostreamers.

Riley \& Luhmann (2012) found that a pseudostreamer in the solar wind during the recent minimum was associated with slow wind. They pointed out that this is not consistent with the empirical relationship often found between magnetic super-radial expansion and solar wind speed (Wang \& Sheeley 1990). Figure 1 (top-middle panel) illustrates this point, i.e. that the field around a bipolar HCS-straddling streamer (streamers in the West and Southeast) expands more than the field around a unipolar pseudostreamer (streamer in the Northeast). Riley \& Luhmann (2012) argued that this supported models where interchange reconnections at a boundary layer between open and closed fields are the source of the slow solar wind, as these predict similar properties for HCS-straddling streamers and pseudostreamers, unlike expansion-based slow wind models.

We cannot tell from our Figure 2 which wind is associated with pseudostreamers. However, we do note that a porcupine-like field by definition has low expansion everywhere, since the "quills" of narrow streamers and surrounding open field lines stick out nearradially. Expansion-factor models would predict that this should lead to faster solar wind overall, and yet 2009 saw the lowest wind velocities at the Earth and Ulysses saw slow and moderate speed wind at mid-latitudes during 2008 and the beginning of 2009.

The temperature of the slow wind is another interesting issue. Arguably for both minima, but particularly for the recent one, the slope in Figure 2 is flatter in the 
slow-wind portion (bottom-left). Indeed, it appears nearly horizontal in the blackdiamonds of the recent minimum. The smaller, narrower loops of the recent minimum may be associated, either directly, or indirectly (e.g., as common consequences of a weakened magnetic field), with the cooler temperatures seen overall. Is it possible that the range in slow-wind temperatures from hot to warm also arises from a range from larger loops to smaller loops? Is it indicative of a time evolution of the size of such loops? Or is it it due to the changing Ulysses orbital position, from HCS-streamer intersecting to higher-latitude pseudostreamers? What is the temperature (and speed) of wind emitting from the small coronal holes at mid-latitude during 2009? These open questions are well worth pursuing, and the recent minimum an excellent opportunity for pinning down the origins and characteristics of the solar wind.

\section{Acknowledgements}

We thank Giuliana de Toma, Larisza Krista, and Alysha Reinard for helpful discussions. We thank Predictive Science Inc. and Janet Kozyra for the material shown in Figure 1, and acknowledge the Ulysses Solar Wind Composition Instrument (SWICS) for solar wind data. LZ is supported by the NASA Living With a Star Heliophysics Postdoctoral Fellowship Program, administered by the University Corporation for Atmospheric Research (NCAR), which is supported by the National Science Foundation.

\section{References}

de Toma, G. 2011, Solar Phys., 274, 195 doi:10.1007/s11207-010-9677-2

Gibson, S. E., Kozyra, J. U., de Toma, G., Emery, B. A., Onsager, T., \& Thompson, B. J. 2009, Journ. Geophys. Res., 114, A09105, doi:10.1029/2009JA014342

Gibson, S. E. et al. 2011, Solar Phys., 274, 5, doi:10.1007/s11207-011-9921-4

Lei, J., Thayer, J. P., Wang, W., \& McPherron, R. L. 2010, Solar Phys., 274, 427, doi:10.1007/s11207-010-9563-y

Mewaldt, R. A. et al. 2010, Astrophys. J. (Letters), 723, L1, 723, doi:10.1088/2041$8205 / 723 / 1 /$ L1

Riley, P. \& Luhmann, J. 2012, Solar Phys., 421, in press, doi:10.1007/s11207-011-9909-0

Thompson, B. et al. 2011, Solar Phys., 274, 29, doi:10.1007/s11207-011-9891-6

von Steiger, R. \& Zurbuchen, T. H. 2011, Journ. Geophys. Res., 116, A01105, doi:10.1029/2010JA015835

Wang, Y.-M. \& Sheeley, N. 1990, Astrophys. J., 355, 726, doi:10.1086/168805

Zhao, L. \& Fisk, L. 2011, Solar Phys., 274, 379, 10.1007/s11207-011-9840-4

\section{Discussion}

JON LINKER: Have you looked at Magnesium in the solar wind?

SARAh Gibson: The $\mathrm{Mg}^{10+} / \mathrm{O}^{6+}$ ratio behaves basically like that of $O^{7+} / \mathrm{O}^{6+}$, in that last minimum there was a bimodal distribution of basically low-speed/high $\mathrm{Mg}^{10+} / \mathrm{O}^{6+}$; high-speed/low $\mathrm{Mg}^{10+} / \mathrm{O}^{6+}$, but this minimum the middle is filled in with middle speed, middle $\mathrm{Mg}^{10+} / \mathrm{O}^{6+}$. Also it is lower amplitude over all speeds, as was true for the $\mathrm{O}^{7+} / \mathrm{O}^{6+}$.

Leif Svalgaard: You shouldn't call it "Heliospheric Current Sheet" so close to the Sun.

JON LINKER: Like it or not, that is what the community calls it. 